\title{
MatOpt: A Python Package for Nanomaterials Design Using Discrete Optimization
}

\author{
${ }_{3}$ Christopher L. Hanselman, Xiangyu Yin, David C. Miller, and Chrysanthos E. Gounaris* \\ Cite This: https://doi.org/10.1021/acs.jcim.1c00984 \\ Read Online
}

4 ABSTRACT: Novel materials are being enabled by advances in 5 synthesis techniques that achieve ever better control over the 6 atomic-scale structure of materials. The pace of materials 7 development has been further increased by high-throughput 8 computational experiments guided by informatics and machine 9 learning. We have previously demonstrated complementary 10 approaches using mathematical optimization models to search 11 through highly combinatorial design spaces of atomic arrange12 ments, guiding the design of nanostructured materials. In this

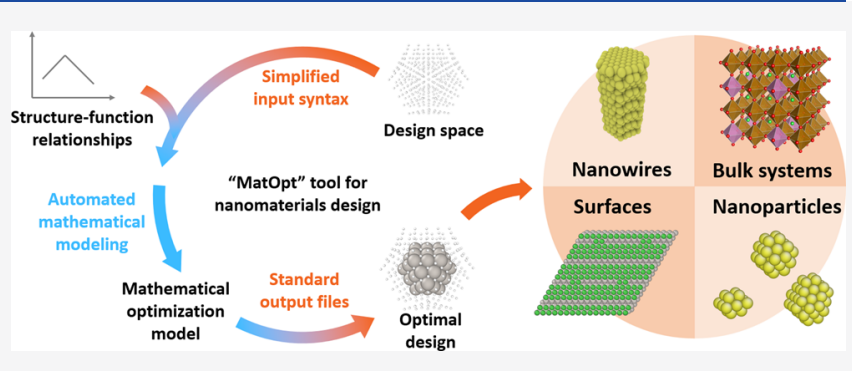
13 paper, we highlight the common features of materials optimization 14 problems that can be efficiently modeled via mixed-integer linear optimization models. To take advantage of these commonalities, 15 we have created MatOpt, a Python package that formalizes the process of representing the design space and formulating optimization 16 models for the on-demand design of nanostructured materials. This tool serves to bridge the gap between practitioners with expertise 17 in materials science and those with expertise in formulating and solving mathematical optimization models, effectively lowering the 18 barriers for applying rigorous numerical optimization capabilities during nanostructured materials development.

\section{$19 \square$ INTRODUCTION}

20 Advances in nanoscale material synthesis are resulting in better 21 control over the atomic-scale structure of materials. ${ }^{1-4}$ 22 Important techniques include the generation of twinned 23 defects, ${ }^{5}$ synthesis of hollow nanostructures, ${ }^{6}$ and tuning of 24 strain, to name but a few. In parallel to synthesis efforts, 25 computational advances in the area of density functional 26 theory (DFT) are enabling the computational design of 27 materials from first-principles. ${ }^{7-9}$ While there are many 28 approaches for controlling structure and predicting properties 29 at the nanoscale, there are relatively few systematic methods 30 for designing nanomaterials algorithmically.

31 Several research efforts take a screening approach to identify 32 highly functional materials by comparing and interpolating in 33 shared databases of experimental and computational re34 sults. $^{10-12}$ Recently, high-throughput computational ap35 proaches and machine learning have been used to massively 36 increase the pace of calculations and to smartly sample the 37 combinatorial materials design space. ${ }^{13-18}$ Another approach 38 has been to develop simplified structure-function relationships 39 for the purpose of predicting material properties as a function 40 of simple geometric descriptors. ${ }^{19,20}$ A notable result can be 41 found in the work of Calle-Vallejo et al., ${ }^{21}$ where the authors 42 used a model for activity as a function of generalized 43 coordination number to design defects on nanostructured 44 surfaces. However, it can be argued that approaches relying on 45 chemist intuition to identify reactive sites might not always 46 provide optimal surfaces, as they lack the ability to extensively 47 search the design space. To improve upon the quality of the resulting structures, Rück et al. ${ }^{22}$ used a similar relationship in 48 conjunction with particle swarm optimization to design 49 symmetric nanoparticles and rods. In the work of Núnez et 50 al., $^{23}$ the authors used another coordination based structure- 51 function model in conjunction with simulated annealing to 52 design defects on nanostructured surfaces. 53

Approaches that leverage metaheuristic optimization meth- 54 ods can find good solutions, but they lack rigorous guarantees 55 on the optimality of the identified designs. In this paper, we 56 seek to identify provably optimal designs by applying exact 57 mathematical optimization algorithms to arrange nanostruc- 58 tured materials from their fundamental building blocks. 59 Previously, we have developed mathematical optimization 60 models for the design of nanostructured surfaces, ${ }^{24,25}$ doped 61 perovskites, ${ }^{26,27}$ metallic nanoclusters, ${ }^{28,29}$ and nanowires. ${ }^{30}$ In 62 each case, we showed how mathematical optimization was 63 well-suited to search the combinatorial design space of 64 nanostructures by formulating appropriate models. 65

Mathematical optimization represents decision problems via 66 variables, algebraic constraints, and an objective function. In 67 eqs 1-4, we express the search for novel materials as a 68

Received: August 12, 2021 
69 mathematical optimization model with generic notation. We 70 denote a vector of variables representing the design as $d$, which 71 must attain values consistent with a design space $D$, and we 72 seek to maximize a desired functionality against several 73 constraints on the stability and fabricability of the design. In 74 this abstract notation, there are no restrictions on the type or 75 scale of materials that can be addressed; however, it is 76 practically important to narrow the scope of problems to focus 77 on cases that are both chemically relevant and that can be 78 tractably modeled. In the next section, we shall discuss some 79 common properties of nanostructured materials and chem80 istries, providing guidance on the subset of systems that can be 81 designed via the below optimization model.

$$
\begin{array}{cl}
\max _{d} & \text { Functionality }(d) \\
\text { s.t. } & \text { Stability }(d) \geq \varepsilon \\
& \text { Fabricability }(d) \geq 0
\end{array}
$$

$d \in \mathcal{D}$

82 The abstract functions referenced in the above materials 83 optimization model can be used to represent a wide variety of 84 functionalities of interest and constraints on the design space, 85 including crystallinity, composition bounds, geometric re86 strictions, thermodynamic relationships, reaction rates, and 87 mass transport, among others. In this context, we should 88 highlight that the ability to solve the resulting optimization 89 models is closely tied to the number and nature of variables 90 and constraints it features, and hence, there is a clear need to 91 make simplifying approximations and carefully match chemical 92 systems to suitable optimization formulations. In general, a 93 modeler will have to properly limit its design efforts to sizes 94 (e.g., unit cells) that strike the right balance between quality of 95 material representation and resulting model tractability. 96 Having said that, it can be argued that solver technology for 97 mixed integer linear programming (MILP) models is more 98 mature compared to that for other model classes, all the while 99 being actively improved at a fast pace. To that end, our 100 proposed design paradigm is largely committed to the casting 101 of linear optimization models, in order to capitalize on the 102 current state-of-the-art and anticipated future improvements of 103 solvers that will effectively enable its application in ever more 104 complex and larger systems.

105 In the remainder of this paper, we shall first describe the 106 common features of material design domains of interest and 107 show how these commonalities result in simple patterns for 108 modeling materials via mathematical optimization. Then, we 109 will describe the implementation of MatOpt, a Python based 110 toolkit for specifying and solving these types of material 111 optimization problems. Finally, we will provide three case 112 studies to illustrate possible use cases of MatOpt for 113 nanoclusters, periodic bulk materials, and catalytic surfaces, 114 identifying the most functional design in each case via the use 115 of well-established numerical optimization software.

\section{COMMON CHARACTERISTICS OF

117 NANOSTRUCTURED MATERIALS

118 Nanostructured materials generally share a few characteristics 119 that lead to the complexity of the design space and, thus, the 120 combinatorial difficulty of finding optimal structures. These 121 common material characteristics provide a basis for defining 122 the scope for our optimization framework and result in simplifying assumptions for our framework to take advantage 123 of. Specifically, we benefit from the discrete nature of lattices, 124 local descriptors of functionality, and periodicity of designs. 125

Discrete Lattices. We generically refer to the individual 126 components of the design as "building blocks." In the example 127 case of nanostructured transition metal catalyst surfaces, the 128 building block can be thought of as an atom, while in larger 129 systems such as metal organic frameworks or supramolecular 130 assemblies, ${ }^{31}$ the building block can be a molecule. 131

Conceptually, one could optimize the design of materials by 132 choosing the Cartesian coordinates for all of the building 133 blocks of interest. However, at the nanoscale, many solid 134 materials tend to form architectures with atoms placed on 135 lattices with specific, discrete locations. While exceptions to 136 this observation can be found (i.e., lattice relaxations around 137 defects, atomic restructuring on nanoclusters), we note that 138 this observation can serve as a good first approximation in 139 many systems of interest. We note that, while we typically work 140 with regular crystalline lattices, our approaches can be applied 141 to other design spaces where the possible placement of matter 142 is ordered but does not satisfy strict definitions of a lattice (i.e., 143 5-fold symmetry around icosahedral nanoclusters). The 144 discrete nature of nanomaterials can let us simplify the search 145 space to only require "yes" or "no" decisions on the choices of 146 building blocks to place in the design. These binary decisions 147 also allow us to preprocess geometric information and avoid 148 encoding nonlinear constraints in our optimization models. 149 For example, as we shall discuss later, we can use piecewise- 150 linear formulations or conformation (pattern) based modeling 151 to encode nonlinear relationships. Reformulated MILP models 152 with binary decision variables are in general expected to be 153 significantly more tractable than their nonlinear counterparts, 154 and thus more amenable to tackle nanomaterials design at a 155 larger scale.

Local Functionality Descriptors. Given a set of discrete 157 locations on which building blocks can be placed, there are also 158 a discrete number of interactions that can contribute to the 159 functionality of a design. In many cases, the desired 160 functionality of the material can be broken down into 161 contributions from sites in the structure. Furthermore, it is 162 also typically the case that the functionality of a site is 163 dependent on the presence of building blocks in a small subset 164 of sites that can be thought of as "neighbors." Strictly speaking, 165 the definition of neighbors does not need to be based on any 166 kind of chemical information (e.g., chemical bonds), nor even 167 on the physical distance between sites, though this is typically a 168 good first approximation. The combination of discrete sites 169 and their neighborhoods (i.e., ordered lists of neighboring 170 sites) leads to the basic data structure that we denote as a 171 "canvas."

The canvas data structure is generically composed of a list of 173 site coordinates in conjunction with a graph, where the latter 174 contains nodes for each site and directed arcs for the neighbor 175 connections. The connections are considered directed because, 176 in general, it is possible for site functionality to depend on each 177 other site asymmetrically. In addition to the standard 178 components of the graph data structure, the canvas specifies 179 an ordering of neighbors to represent specific types of 180 connections. This is useful for the ability to represent the 181 specific alignment of neighbors in the lattice, which is 182 necessary when trying to indicate a particular configuration 183 of atoms in a design. In Figure 1, we present an example canvas $184 \mathrm{fl}$ with the corresponding data structure for the site neighbor- 185 


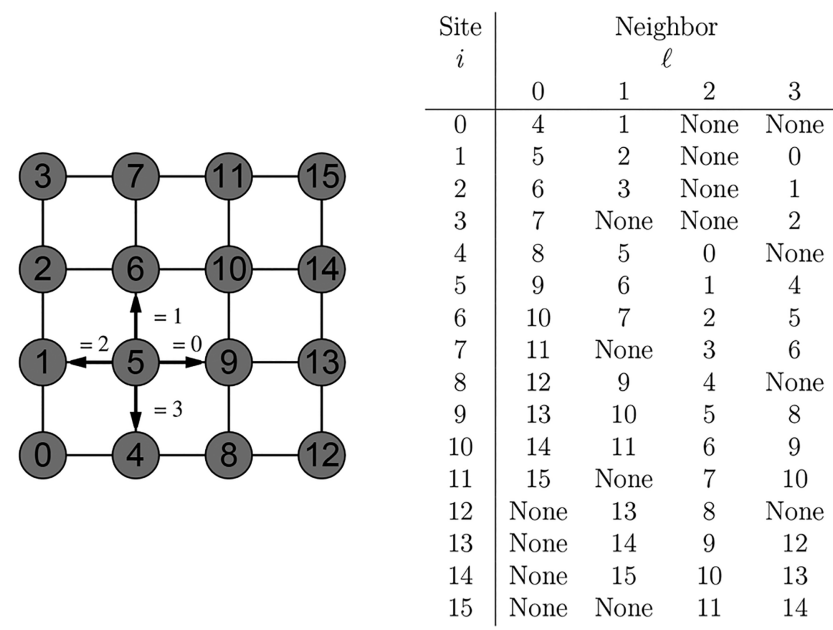

Figure 1. Example ordered neighbor connections for a simple canvas. (left) Graphical representation depicting connections between the 16 sites indexed from 0 to 15 ; the concept of neighbors is illustrated with site 5 , where the arrows point to its four neighbors in four directions indexed from 0 to 3. (right) Tabular representation for use in code.

186 hoods. In this example, the ordering of neighbors consistently 187 corresponds to sites along the four cardinal directions.

188 Material Periodicity. Nanostructured materials are 189 modeled via cells containing periodicity in $0,1,2$, or 3 190 dimensions, corresponding to clusters, wires, surfaces, and bulk 191 materials, respectively. From an implementation standpoint, 192 the presence of periodicity can lead to nonintuitive 193 neighborhoods with connections that cross periodic bounda194 ries. These connections can be identified from the combination 195 of the canvas sites with a set of rules for transforming points 196 that cross the tiling boundaries. Given a fixed canvas, these 197 connections can be tabulated prior to formulating a 198 mathematical optimization model, while care should be taken 199 to ensure that the canvas neighborhoods are geometrically 200 consistent. Figure 2 illustrates a simple two-dimensional lattice 201 with connections defined by the periodicity of the canvas. In 202 this example, the shape of the periodic tile is denoted with the

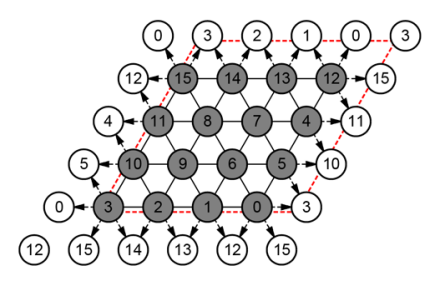

\begin{tabular}{c|cccccc}
$\begin{array}{c}\text { Site } \\
i\end{array}$ & \multicolumn{7}{|c}{$\begin{array}{c}\text { Neighbor } \\
\ell\end{array}$} \\
& E & NE & NW & W & SW & SE \\
\hline 0 & 3 & 5 & 6 & 1 & 12 & 15 \\
1 & 0 & 6 & 9 & 2 & 13 & 12 \\
2 & 1 & 9 & 10 & 3 & 14 & 13 \\
3 & 2 & 20 & 5 & 0 & 15 & 14 \\
4 & 11 & 12 & 13 & 7 & 5 & 10 \\
5 & 10 & 4 & 7 & 6 & 0 & 3 \\
6 & 5 & 7 & 8 & 9 & 1 & 0 \\
7 & 4 & 13 & 14 & 8 & 6 & 5 \\
8 & 7 & 14 & 15 & 11 & 9 & 6 \\
9 & 6 & 8 & 11 & 10 & 2 & 1 \\
10 & 9 & 11 & 4 & 5 & 3 & 2 \\
11 & 8 & 15 & 12 & 4 & 10 & 9 \\
12 & 15 & 0 & 1 & 13 & 4 & 11 \\
13 & 12 & 1 & 2 & 14 & 7 & 4 \\
14 & 13 & 2 & 3 & 15 & 8 & 7 \\
15 & 14 & 3 & 0 & 12 & 11 & 8
\end{tabular}

Figure 2. Example neighbor connections across the boundary of a periodically tiled canvas. (left) Graphical representation depicting connections between the 16 sites indexed from 0 to 15 and their periodic counterparts; connections across the periodic boundary (red dashed line) are shown with arrows. (right) Tabular representation for use in code. dashed red parallelogram and neighbors crossing the tile edge 203 are shown with directed arcs.

It is important to highlight that the size of a periodic canvas 205 constrains the design space; hence, it might lead the resulting 206 optimization model to find an "optimal" solution but miss 207 better designs that are only possible with a larger repeating 208 pattern. To that end, in the case of periodic designs, a natural 209 algorithm for quickly identifying good solutions is to iteratively 210 expand the size of the periodic canvas and solve again until no 211 further improvements are found with increasing size, assuming 212 the available computational resources are not exceeded in the 213 process. While we may not know the truly optimal solution a 214 priori, a typical observation would be to see the same design 215 repeated, as optimal solutions are obtained under consecutively 216 increasing canvas sizes that are consistent with the periodicity 217 of the design.

218

\section{MODELING ELEMENTS}

219

Given the commonalities in the materials of interest, there are 220 several optimization modeling patterns that we can use to 221 modularly formulate models to be used for rigorous design. In 222 particular, the discrete nature of the design space leads to 223 formulations that naturally employ binary variables. Further- 224 more, as we will demonstrate in our case studies, there are 225 several strategies to exactly encode information via linear 226 constraints and implication logic, even in cases where complex 227 or nonlinear structure-function relationships are of interest. 228

Model Variables. The common features of nanostructured 229 materials leads to a natural set of variables from which 230 mathematical optimization models can be formulated. Below, 231 we present four types of basic variables in addition to several 232 types of auxiliary variables representing their aggregation. 233

Presence of Building Blocks. The presence of a building 234 block of a given type at a particular site is the most 235 fundamental variable in the optimization model. In eq 5, we 236 denote as $\tilde{Y}_{i k}$ the presence of a building block of type $k$ in site $i .237$ We denote the set of sites in the canvas as $I$ and the set of 238 building blocks as $K$.

239

$$
\tilde{Y}_{i k} \in\{0,1\} \quad \forall k \in K, \forall i \in I
$$

Presence of Bonds. Given the variables for the presence of 241 specific building block types, we can encode information about 242 the connections between building blocks. For convenience, we 243 refer to these variables as "bond" type variables, but in general, 244 they do not have to correspond to bonds in the chemical sense. 245 In eqs 6-9, we denote as $\tilde{X}_{i j k l}$ the presence of a bond between 246 a building block of type $k$ at site $i$ and a building block of type $l 247$ at site $j$. In the below, $N_{i}$ denotes the set of neighboring sites to 248 location $i$.

$$
\tilde{X}_{i j k l} \leq \tilde{Y}_{i k} \quad \forall l \in K, \forall k \in K, \forall j \in N_{i}, \forall i \in I
$$$$
\tilde{X}_{i j k l} \leq \tilde{Y}_{j l} \quad \forall l \in K, \forall k \in K, \forall j \in N_{i}, \forall i \in I
$$

$$
\begin{aligned}
& \tilde{X}_{i j k l} \geq \tilde{Y}_{i k}+\tilde{Y}_{j l}-1 \\
& \forall l \in K, \forall k \in K, \forall j \in N_{i}, \forall i \in I \\
& \tilde{X}_{i j k l} \in\{0,1\} \\
& \quad \forall l \in K, \forall k \in K, \forall j \in N_{i}, \forall i \in I
\end{aligned}
$$


254 Neighbor Counts. Applications at the nanoscale often 255 correlate functionality to counts of neighbors around a 256 particular type of site. In general, this functionality can also 257 be broken down into contributions from sites and neighbors of 258 particular types. In eqs $10-11$, we denote as $\tilde{C}_{i k l}$ the count of 259 bonds between a building block of type $k$ at site $i$ and 260 neighboring building blocks of type $l$. In this definition, if there 261 is not a building block of type $k$ at site $i$, then all $\tilde{C}_{i k l}$ counts are 262 set to zero.

263

$$
\tilde{C}_{i k l}=\sum_{j \in N_{i}} \tilde{X}_{i j k l} \quad \forall l \in K, \forall k \in K, \forall i \in I
$$$$
\tilde{C}_{i k l} \in\left\{0,1, \ldots,\left|N_{i}\right|\right\} \quad \forall l \in K, \forall k \in K, \forall i \in I
$$

265 The domain of variables $\tilde{C}_{i k l}$ could in principle be 266 equivalently relaxed to the continuous interval $\left[0,\left|N_{i}\right|\right]$, since 267 the integrality of the variables is enforced by eqs 10 . For 268 example, this may be advantageous when one wants to limit 269 the number of integer variables (as opposed to continuous 270 ones) in the overall formulation. However, our computational 271 investigations suggest that most often the integer variable 272 definition results in a more tractable optimization model, 273 something that could be attributed to the fact that the branch274 and-bound type algorithm employed in the solver can in this 275 case exploit those variables during branching. To that end, the 276 "default" MatOpt implementation of $\tilde{C}_{i k l}$ variables is to define 277 them internally as integer variables.

278 Aggregate Variables. Variables with type-dependent 279 information may not always be necessary or useful for 280 modeling a given system. For example, many descriptors are 281 expressed with conditionals such as the presence of "any atom" 282 or "any bond" being present in the material. For each of the 283 type-dependent variables previously discussed, we also present 284 aggregated versions that indicate the presence of any building 285 block or bond, or the count of neighbors. In eqs 12-21 we 286 denote as $Y_{i}$ the presence of any building block at site $i$, we 287 denote as $X_{i j}$ the presence of any type of bond between sites $i$ 288 and $j$, and we denote as $C_{i}$ the count of any type of neighbor 289 next to any type of building block present at site $i$. Similarly to 290 variables $\tilde{C}_{i k l}$, variables $C_{i}$ can be relaxed to continuous 291 variables attaining values in their corresponding continuous 292 interval.

$$
\begin{aligned}
& 293 \quad Y_{i} \in\{0,1\} \quad \forall i \in I \\
& 294 \quad X_{i j} \leq Y_{i} \quad \forall j \in N_{i}, \forall i \in I \\
& { }_{295} \quad X_{i j} \leq Y_{j} \quad \forall j \in N_{i} \forall i \in I \\
& 296 \quad X_{i j} \geq Y_{i}+Y_{j}-1 \quad \forall j \in N_{i}, \forall i \in I \\
& 297 \quad X_{i j} \in\{0,1\} \quad \forall j \in N_{i}, \forall i \in I \\
& C_{i}=\sum_{j \in N_{i}} X_{i j} \quad \forall i \in I \\
& 299 \quad C_{i} \in\left\{0,1, \ldots,\left|N_{i}\right|\right\} \quad \forall i \in I \\
& 300 \\
& Y_{i}=\sum_{k \in K} \tilde{Y}_{i k} \quad \forall i \in I \\
& 301 \\
& X_{i j}=\sum_{k \in K} \sum_{l \in K} \tilde{X}_{i j k l} \quad \forall j \in N_{i}, \forall i \in I
\end{aligned}
$$

$$
C_{i}=\sum_{k \in K} \sum_{l \in K} \tilde{C}_{i k l} \quad \forall i \in I
$$

Presence of Conformations. In addition to variables for 303 indicating bonds and neighbor counts, we also encode 304 variables for indicating specific combinations of neighbors, 305 a.k.a. "conformations." In eqs $22-25$, we denote with $\tilde{Z}_{i c}$ the 306 presence of a conformation of type $c$ at site $i$. We use the 307 parameter $\xi_{i c j l}$ to indicate if a conformation of type $c$ that is 308 located at site $i$ should feature a building block of type $l$ in 309 neighboring location $j$.

$$
\begin{aligned}
& \tilde{Z}_{i c} \leq \tilde{Y}_{j l} \\
& \forall l \in K:\left\{\xi_{i c j l}=1\right\}, \forall j \in N_{i}, \\
& \forall c \in C, \forall i \in I \\
& \text { (22) } 311 \\
& \tilde{Z}_{i c} \leq 1-\tilde{Y}_{j l} \\
& \forall l \in K:\left\{\xi_{i c j l}=0\right\}, \forall j \in N_{i}, \\
& \forall c \in C, \forall i \in I \\
& \tilde{Z}_{i c} \geq 1-\sum_{j \in N_{i}} \sum_{\substack{l \in K: \\
\left\{\xi_{i c j}=1\right\}}}\left(1-\tilde{Y}_{j l}\right)-\sum_{j \in N_{i}} \sum_{\substack{l \in K: \\
\left\{\xi_{i c j}=0\right\}}} \tilde{Y}_{j l} \\
& \forall c \in C, \forall i \in I \\
& \tilde{Z}_{i c} \in\{0,1\} \quad \forall c \in C, \forall i \in I
\end{aligned}
$$

Common Constraint Patterns. Given the various sets of 315 model variables introduced above, one may define a wide 316 variety of additional, application-specific material descriptors, 317 which can later be utilized to impose restrictions on the design 318 space. In this section, we provide several basic patterns of 319 constraints for defining new descriptors. In each case, we 320 present a few basic patterns, noting that there are many ways to 321 apply these patterns for combinations of site and bond types. 322

Linear and Piecewise-Linear Constraints. The most 323 straightforward constraints to incorporate in MILP models 324 are simple linear equalities or inequalities. In our models, these 325 often take the form of budget constraints or summations. 326 Similarly, we can incorporate piecewise-linear expressions into 327 the model by introducing additional binary variables and 328 implication logic.

Because the basic decision variables are discrete, all 330 subsequent variables also attain discrete value. Therefore, we 331 can practically consider piecewise-linear constraints to 332 incorporate any nonlinear function into material design models 333 without introducing approximation error. We achieve this by 334 encoding breakpoints to coincide with the discrete feasible 335 points, as illustrated in Figure 3. Assuming the number of $336 \mathrm{f} 3$ required breakpoints is not too large, this technique can be 337 used to exactly encode nonlinear functions without sacrificing 338 accuracy. In other cases, it may still be necessary to estimate 339 the original function to maintain computational tractability. In 340 eqs 26-27, we present the general notation for representing 341 simple constraints, where $g(x)$ and $P(x)$ denote linear and 342 piecewise-linear expressions, respectively. Here, the vector $x$ is 343 used to generically represent the variables and parameters used 344 in the expression. Also note that, although eqs $26-27$ are cast 345 here as $\leq 0$ constraints, one may readily model any 346 combination of $=, \geq$, and nonzero right-hand sides.

$$
\begin{aligned}
& g(x) \leq 0 \\
& P(x) \leq 0
\end{aligned}
$$




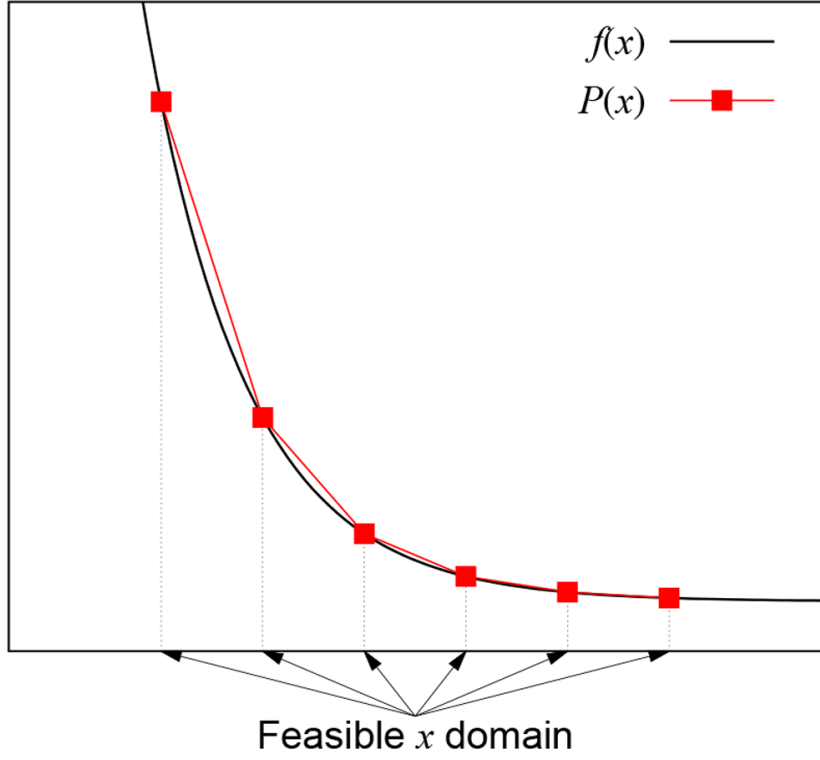

Figure 3. Example piecewise-linear function to exactly encode a nonlinear function over a discrete domain.

350 Implication Constraints. Frequently, material descriptors 351 are defined in terms of logical predicates or conclusions. In 352 such cases, we can use implication logic to conditionally apply 353 constraints on the design space. We identify three general 354 patterns of logical implications that are encountered in material 355 design optimization models. In each case, a linear expression is 356 conditionally set to zero if a binary indicator variable is active 357 (i.e., equal to 1). Note that some predefined model variable ${ }_{358}$ (e.g., $\tilde{Y}_{i k}, \tilde{X}_{i j k l}, \tilde{Z}_{i c}$ ), or some user-defined descriptor, will serve 359 as the indicator variable (generically denoted below as $D_{(\cdot)}$ ) in 360 each case.

361 Site and Bond Descriptor Implications. The first general 362 type of logical constraint forces an expression to be zero if a 363 given condition is true for the site. In eqs 28-29, $g_{i}(x)$ 364 corresponds to a general linear expression that is required to be 365 equal to 0 if binary indicator $D_{i}$ is equal to 1 at site $i$. The 366 parameters $M^{\mathrm{LB}}$ and $M^{\mathrm{UB}}$ are so-called "big-M" parameters that 367 can be automatically calculated to correctly encode the design 368 space in the case that the binary indicator is inactive. The 369 conditional expression can also be negated so that an 370 expression is forced to be zero if the condition is not met. 371 An example of invoking this pattern is the case when one 372 requires a site's generalized coordination number to be equal 373 to a target value if the site is considered a reactive site, and 374 equal to zero otherwise.

$$
M^{\mathrm{LB}}\left(1-D_{i}\right) \leq g_{i}(x) \leq M^{\mathrm{UB}}\left(1-D_{i}\right) \quad \forall i \in I
$$

375

$$
376 \quad D_{i} \in\{0,1\} \quad \forall i \in I
$$

377 Similarly, logical implication constraints can be written 378 across the bonds in the canvas, where the linear expression and 379 associated indicator variables are suitably indexed over 380 neighbor location pairs, as eqs 30-31 show. An example of 381 this pattern is requiring the binding energy to be equal to an 382 expression only if a bond is actually present between two sites.

$$
\begin{aligned}
& M^{\mathrm{LB}}\left(1-D_{i j}\right) \leq g_{i j}(x) \leq M^{\mathrm{UB}}\left(1-D_{i j}\right) \\
& \quad \forall j \in N_{i}, \forall i \in I \\
& D_{i j} \in\{0,1\} \quad \forall j \in N_{i}, \forall i \in I
\end{aligned}
$$

Site Combination Implications. The second pattern of 385 logical implications involves combinations of neighboring sites. 386 In this case, while the linear expressions of interest are 387 location-specific, the indicator variables are indexed over the 388 set of neighbor pairs in a canvas to create individual constraints 389 on each of the sites. This pattern is used to encode the basic 390 variables for the presence of bonds and can also be used in 391 user-defined descriptors for encoding neighboring pairs of 392 conformations.

$$
\begin{aligned}
& M^{\mathrm{LB}}\left(1-D_{i j}\right) \leq g_{i}(x) \leq M^{\mathrm{UB}}\left(1-D_{i j}\right) \\
& \quad \forall j \in N_{i}, \forall i \in I \\
& M^{\mathrm{LB}}\left(1-D_{i j}\right) \leq g_{j}(x) \leq M^{\mathrm{UB}}\left(1-D_{i j}\right) \\
& \quad \forall j \in N_{i}, \forall i \in I \\
& D_{i j} \in\{0,1\} \quad \forall j \in N_{i}, \forall i \in I
\end{aligned}
$$

Neighborhood Implications. The third pattern of logical 397 implications introduces constraints on all neighboring sites to a 398 location in the canvas. This pattern is used to encode the basic 399 variables for the presence of conformations and is also 400 generally useful for introducing more complicated constraints 401 on combinations of sites in the canvas.

402

$$
\begin{aligned}
& M^{\mathrm{LB}}\left(1-D_{i}\right) \leq g_{j}(x) \leq M^{\mathrm{UB}}\left(1-D_{i}\right) \quad \forall j \in N_{i}, \forall i \in I \\
& D_{i} \in\{0,1\} \quad \forall i \in I
\end{aligned}
$$

\section{THE MATOPT TOOLKIT}

To assist researchers in casting and solving rigorous 406 mathematical optimization models for nanostructured materi- 407 als design, we have developed MatOpt, an object-oriented 408 Python based library that implements the modeling framework 409 discussed in the previous section. This toolkit formalizes and 410 simplifies the process for carrying out nanostructured materials 411 optimization via two major contributions. First, we provide 412 several modeling objects for specifying the materials design 413 space from simple input. Second, we provide a framework for 414 specifying structure-function relationships without needing a 415 detailed understanding of how that specification can be 416 converted into a suitable mathematical optimization model. 417 While the explanation given here is kept at a high level, we 418 encourage the interested reader to refer to the detailed 419 documentation and open-source code available online as part 420 of the Institute for the Design of Advanced Energy Systems 421 (IDAES) code distribution. ${ }^{32}$

Software Availability and Solver Dependencies. 423 MatOpt is bundled with the open-source IDAES PSE 424 framework $^{33}$ and can be downloaded freely at https://github. 425 com/IDAES/idaes-pse/tree/main/idaes/apps/matopt. Users 426 should follow the instructions described in the online 427 documentation $^{34}$ to install and access the toolkit. Along with 428 the source code, we also provide several use cases in the form 429 of Jupyter notebooks, ${ }^{35}$ which demonstrate the features and 430 


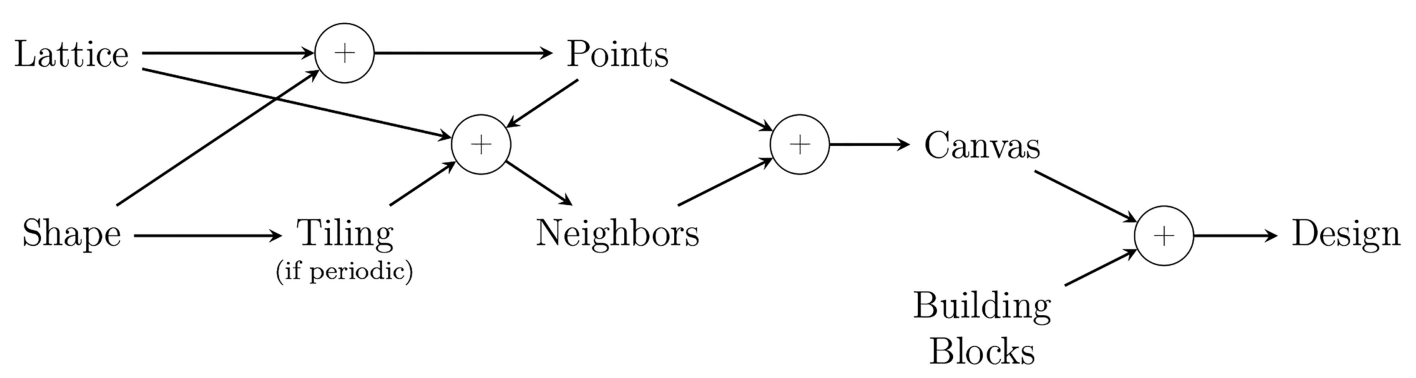

Figure 4. Flow of information for creating objects to represent materials.

431 functionalities of the toolkit as well as offer useful Python 432 scripts from which users can start to develop new codes for 433 their own materials design applications. The scripts and source 434 code can be downloaded from the IDAES example repository 435 at https://github.com/IDAES/examples-pse/tree/main/src/ 436 Examples/MatOpt.

437 At its core, MatOpt translates a materials optimization 438 problem into a Python Optimization Modeling Objects 439 (Pyomo) model object that can then be solved by an available 440 MILP solver, with MatOpt providing appropriate shortcut 441 functions to facilitate this step. Experienced Pyomo users with 442 access to a licensed MILP solver can easily select their solver of 443 choice during this process. For users who either do not have 444 such access or are not familiar with how to set up and use 445 optimization solvers in Pyomo, MatOpt further automates the 446 invocation of the web based free solver NEOS-CPLEX that can 447 be utilized for this purpose. ${ }^{36-38}$

448 Materials Representation Objects. The focus of this 449 section is to describe the ability of the toolkit to easily 450 instantiate data structures necessary to represent nanoscale 451 materials from simple input. Figure 4 illustrates the interactions 452 between several objects and data structures to represent 453 nanostructured material designs in MatOpt. The basic data 454 structure required to cast an optimization model is a Canvas, 455 which is essentially a list of Cartesian points coupled with a 456 graph, whose nodes correspond to sites and whose arcs 457 correspond to bonds. This object establishes a mapping from 458 the abstract mathematical modeling of materials as graphs to 459 the geometry of the material's lattice.

460 The list of points necessary to create a Canvas can be 461 obtained from the combination of a Lattice and a Shape 462 object by scanning over the set of lattice sites that fall inside 463 the shape. The set of neighbor connections to be encoded in 464 the Canvas can be identified from rules that are attached to 465 the Lattice object. In the simplest case, these rules for 466 indicating neighbors can be simple cutoff distances, but we also 467 note cases where asymmetric definitions of neighbors can be 468 useful for representing materials. For example, in Hanselman et $469 \mathrm{al}^{2},{ }^{27}$ the authors utilized an asymmetric definition of 470 neighborhoods to establish different definitions of neighbors 471 around oxygen atoms compared to metallic B-site atoms. 472 Complementary to the rules defined by the lattice, a Tiling 473 object can be used to make the neighbor connections 474 consistent across the boundary of a periodic tile.

475 While a Canvas object holds topological and geometric 476 information, a Design object is composed of a Canvas in 477 conjunction with a list of building blocks placed in the sites of 478 the material. The necessary attributes of a building block are 479 encoded in the BuildingBlock class and can in principle 480 be extended to represent many materials. As a starting point, we have implemented the Atom class that is of interest in our 481 work.

It is worthy to note that several existing tools provide 483 interfaces for setting up and analyzing materials at the atomic 484 scale. $^{39-41}$ Their emphasis is on setting up computational 485 experiments that can efficiently calculate material properties 486 during atomic-scale simulations. Although these tools typically 487 include optimization routines, the latter serve to minimize the 488 energy of atomic configurations by relaxing the atomic 489 coordinates in the vicinity of lattice sites; in this sense, their 490 use of optimization is conceptually different from the design 491 paradigm discussed in this work. Furthermore, these codes are 492 tailored to work with more traditional workflows in computa- 493 tional materials screening and, therefore, do not immediately 494 adapt to the same level of generality that the mathematical 495 optimization approach can exhibit. Whereas MatOpt's basic 496 data structures were defined to support the representation of 497 the materials design space at the level of detail required by the 498 various transition metallic systems we wanted to contemplate, 499 there exist opportunities for extending these language features. 500 Future versions could extend MatOpt by incorporating 501 connections between other open-source packages and our 502 optimization modules.

Algebraic Modeling Language Features. While the 504 goal of MatOpt's material modeling modules is to help 505 organize and create prerequisite data structures, the goal of the 506 optimization modules is to enable users to cast optimization 507 models from minimal input. One of the key features of our 508 modeling approach is the automatic encoding of logical 509 expressions into MILP models. Additionally, we automatically 510 generate indexed expressions for a wide variety of patterns 511 encountered in material optimization models. In this way, our 512 tool can be used by practitioners without a background in 513 mathematical optimization and from simplified user input. 514 Conversely, a user who is already familiar with Pyomo can use 515 MatOpt to generate a Pyomo model with a partial formulation 516 defined and then modify it as necessary (e.g., by adding 517 customized constraints).

Given a defined Canvas and a list of BuildingBlock 519 objects to place in the design, we start by automatically 520 generating the set of basic descriptors for the presence of 521 building blocks, bonds, neighbor counts, and conformations. 522 We note that MatOpt will infer from user inputs what basic 523 descriptors are needed, generating and indexing constraints 524 accordingly, but there is no guarantee that MatOpt will identify 525 all possible simplifications and return the optimization model 526 that is most simplified in this regard. In principle, however, 527 power users can implement further simplifications by 528 modifying the resulting Pyomo model directly. Beyond these 529 basic descriptors, MatOpt enables users to specify additional 530 descriptors using a combination of Expression and Rule 531 
532 objects. The general flow of information for specifying a model 533 is presented in Figure 5. The Expression objects are

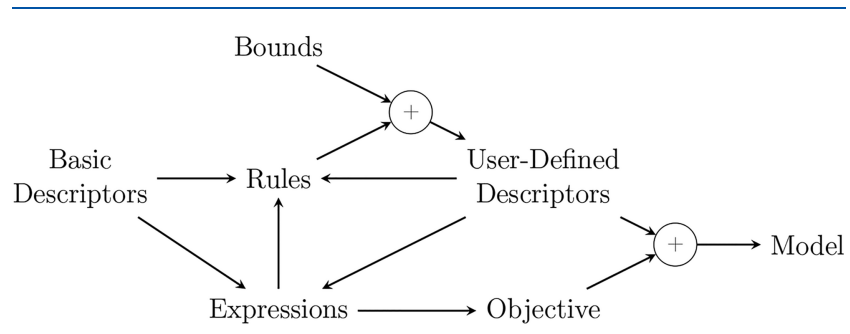

Figure 5. Flow of information for instantiating MatOpt optimization models.

534 generated from basic or user-defined descriptors via a set of 535 predefined patterns. Two examples include SumSites and 536 SumBonds to allow simple summations. A more complicated 537 example is SiteCombination which creates expressions 538 indexed over bonds by adding contributions from descriptors 539 at two neighboring sites. The Expression objects are used 540 in conjunction with Rule objects to define descriptors and to 541 generate constraints on the design space without requiring the 542 user to specify the details of the underlying constraints or 543 necessary reformulations. A full list of available expressions and 544 descriptor rules, along with some example use cases, can be 545 found in Section A of the Supporting Information, with the 546 expectation that additional patterns can be introduced as part 547 of ongoing open-source development.

548 When the user requests to optimize the model, the code will 549 automatically interpret Rule objects to generate a Pyomo 550 optimization model. ${ }^{42,43}$ Several rules, such as LessThan, 551 EqualTo, and GreaterThan, can be immediately 552 interpreted as linear constraints in the model. Other rules 553 might require some more specialized treatment. For example, 554 the Implies rule creates implication constraints that are 555 enforced only if the site descriptor is true (e.g., if a site is 556 present, if a bond is present, etc.). The FixedTo rule is 557 included to allow descriptors to be explicitly fixed to a value. 558 While, in principle, the same effect could be achieved by 559 introducing equality constraints into the model, the FixedTo 560 routines have the additional efficiency of fixing logically 561 implied basic variables when possible. The Piecewise562 Linear rule allows the user to specify the variable domain, 563 values, and breakpoints to equate a descriptor to a piecewise linear function. When generating constraints, MatOpt converts 564 PiecewiseLinear rules to specialized objects in Pyomo. 565 In general, this leverages advances in Pyomo and its supported 566 optimization solvers and enables interested users to quickly try 567 several alternate mathematical reformulations of piecewise 568 functions provided by Pyomo.

One of the useful features of our modeling framework is the 570 automatic handling of expression and rule indexing. For 571 example, if a user-defined variable is indexed over a subset of 572 sites in the canvas (e.g., over only the oxygen sites in a lattice), 573 then the derived expressions and constraints are likewise 574 indexed over that subset. Alternatively, if a variable indexed 575 over canvas sites is multiplied by a parameter that is indexed 576 over site types, then the resulting expression is automatically 577 indexed over the set product of sites with site types. Figure $6578 \mathrm{ff}$ presents an example descriptor definition and highlights the 579 data structures for indexing that are maintained for each 580 descriptor, expression, and rule in the model. As an example 581 descriptor, we present an abstract structure-function relation- 582 ship that assigns the pairwise binding energy $\left(B E_{i j}, 583\right.$ programmatically $\mathrm{m} . \mathrm{BE} i j)$ to an expression formed from a 584 weighted combination of the coordination number $\left(C_{i}, 585\right.$ programmatically $\mathrm{m} . \mathrm{Ci}$ ) conditionally on the type of binding 586 that is present $\left(\tilde{X}_{i j k l}\right.$, programmatically m.Xijkl). The ${ }_{587}$ constraint includes bond type-indexed parameters $\left(A_{k l}, 588\right.$ programmatically $\mathrm{m} . \mathrm{Akl}$ ) that abstractly represent the 589 weighting contributions.

590

The last component necessary to specify an optimization 591 model is a single expression to minimize or maximize as an 592 objective function. While optimization solvers typically only 593 accept a single objective, we note that it is possible to combine 594 multiple objectives via a weighted sum implemented as a 595 LinearExpr object. One of our case studies in the next 596 section exemplifies this and illustrates how optimization 597 models can be used to generate Pareto-optimal frontiers. $\quad 598$

\section{CASE STUDIES}

599

As a demonstration of the MatOpt framework, we present 600 three example case studies. In the first example, we build a 601 simple nanocluster stability optimization model using minimal 602 programming effort. This example illustrates the basic 603 workflow and simplified input syntax, exemplifying how 604 MatOpt can lower the barriers to apply rigorous mathematical 605 optimization in the area of nanostructrured materials design. In 606

m.Xijkl.rules.append(Implies ((m.BEij,EqualTo(SiteCombination(m.Ci,m.Akl))))

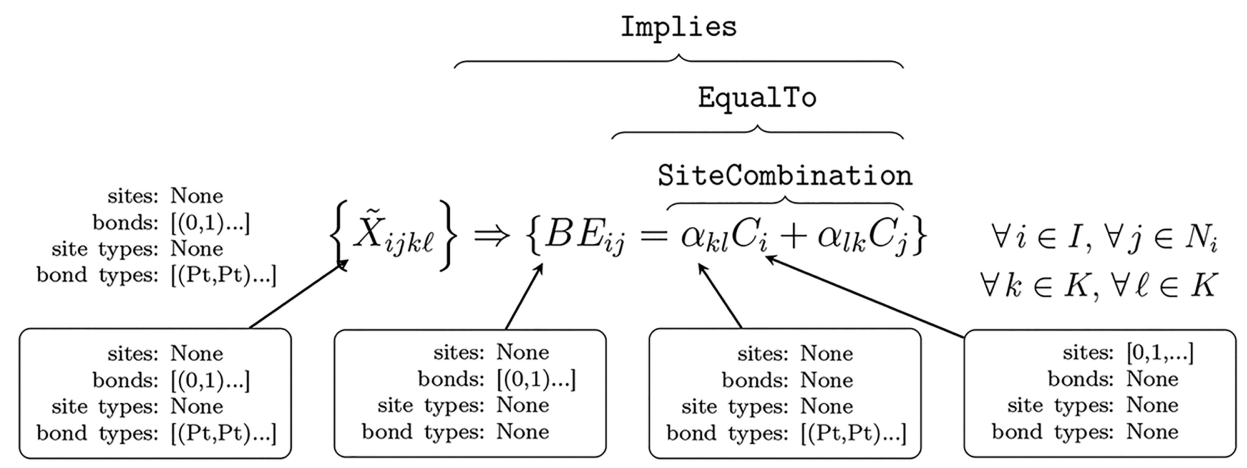

Figure 6. Example creation of site descriptor rules. Example code is given in the top row, and the equivalent mathematical notation is given in the middle. Attributes implied by automatic indexing are presented in the bottom row. 


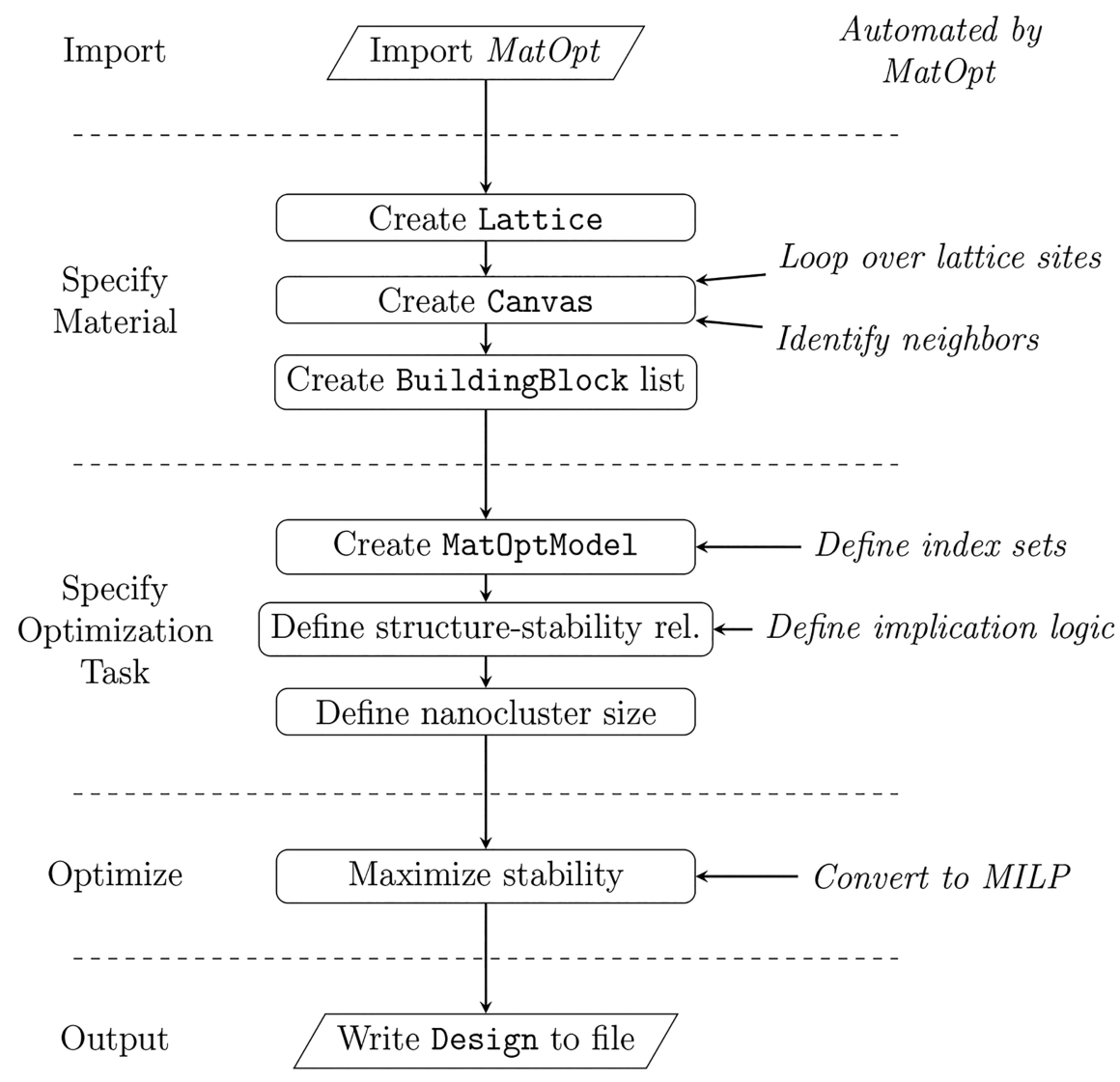

Figure 7. Conceptual flowchart for the nanocluster cohesive energy example.

607 the second example, we identify the best placement of dopants 608 in bulk perovskite materials for optimal oxygen diffusivity. We 609 introduce syntax to deal with unit cell periodicity and 610 conformation based design. This example also showcases 611 how DFT calculations can inform a mathematical optimization 612 model in the context of a materials design problem. In the 613 third example, we show how MatOpt can be employed to 614 design a bifunctional catalyst surface, targeting both reactivity 615 and stability, in the context of a system studied recently in the 616 literature. ${ }^{44}$ This example further demonstrates two-dimen617 sional periodicity syntax and the flexibility of modeling a more 618 complex multiobjective problem with only a modest increase in 619 programming effort.

620 Cohesive Nanocluster Design. The recent work of 621 Isenberg et al. ${ }^{28}$ demonstrated the use of mathematical 622 optimization models to identify cohesive nanoclusters via a 623 set of tailored algorithms. Here, we present a simplified version 624 of their models as an example system on which MatOpt can be 625 employed. As the structure-stability relationship, we consider 626 a normalized version of the Tománek model for cohesive 627 energy $^{45}$ shown in eq 37 . Here, $\hat{E}^{\text {coh }}$ corresponds to the 628 normalized cohesive energy of a nanocluster, $C N_{\text {bulk }}$ is the 629 material's bulk coordination number, $N$ is the size of the 630 nanocluster, and $C_{i}$ corresponds to the coordination number of 631 atom $i$.

632

$$
\hat{E}^{\text {coh }}=\frac{1}{N \sqrt{C N_{\text {bulk }}}} \sum_{i \in I} \sqrt{C_{i}}
$$

633 Figure 7 presents the conceptual steps that we will be 634 following to specify this nanocluster energy minimization problem; that is, importing the MatOpt module, providing 635 input about the materials system of interest, specifying the 636 relevant structure-function and constraint information, 637 invoking the optimizer, and finally, outputting the identified 638 designs.

In the following section, we present the minimal code 640 necessary to set up and solve the nanocluster cohesive energy 641 optimization problem. First, we import the MatOpt package 642 and standard Python modules.

$643 \mathrm{~g}$

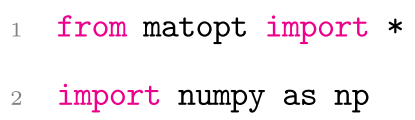

Next, we define the objects necessary to represent the 644 material information in the problem. For this, we create a 645 Lattice object that specifies the sites to consider in the 646 design space along with their neighbor connections. In this 647 case, the object will codify information pertaining to a face- 648 centered cubic (FCC) lattice of a specific interatomic distance. $649 \mathrm{~g}$

3 Lat $=$ FCCLattice $($ IAD $=2.77)$

In the next few lines of code, we build a Canvas object 650 composed of three shells of FCC lattice locations around the 651 origin. The particular choice of three shells was based on the 652 original work of Isenberg et al., ${ }^{28}$ where an adaptive canvas 653 resizing technique was used to identify the best canvas size. As 654 discussed, it is important for users to carefully choose the size 655 of the canvas so as to properly navigate the trade-off between 656 global optimality and model tractability. Note that, since we 657 are using a lattice defined in three-dimensional space, the 658 
659 expected data type of a point is a $\mathrm{NumPy}^{46}$ array of length 660 three with floating point precision.

\author{
Canv $=\operatorname{Canvas}()$ \\ Canv.addLocation(np.array $([0,0,0]$, dtype $=f l o a t))$ \\ 6 Canv.addShells (3, Lat.getNeighbors)
}

661 In the following couple of lines, we define a parameter, $\mathrm{N}$, to 662 be the number of building blocks to consider in the 663 nanocluster; in this case, 17 atoms. Additionally, we define 664 the set of Atom objects to place in the design, specifying their 665 elemental identify as gold atoms, for convenience.

$$
\begin{aligned}
& { }_{7} \mathrm{~N}=17 \\
& 8 \text { Atoms }=\text { [Atom('Au')] }
\end{aligned}
$$

666 Once the material information is specified, we can begin to 667 generate a mathematical optimization model via an object of 668 class MatOptModel. The model object is initialized from a 669 Canvas and a list of Atom objects from which we can 670 identify the applicable index sets for sites, bonds, site types, 671 and bond types. These index sets automatically used as new 672 descriptors are defined.

$$
9 \mathrm{~m}=\text { MatOptModel (Canv, Atoms) }
$$

673 In the following steps, we introduce material descriptors to 674 encode the structure-stability relationship as well as to 675 constrain the design space of interest. First, we introduce a 676 descriptor for the square root of the coordination number. 677 Because the coordination number is itself discrete, we can 678 encode its square root without approximation error via a 679 piecewise linear formulation, thereby preserving the linearity of 680 the model. Note that, since the input descriptor (i.e., the 681 coordination number $\mathrm{m} . \mathrm{Ci}$ ) is indexed over all sites, the 682 resulting rule and descriptor are also indexed over all sites. 683 Therefore, we utilize the method addSitesDescriptor, 684 which registers the new user-defined descriptor with the 685 model.

$10 \mathrm{~m}$.addSitesDescriptor (' $\mathrm{CNRi}$ ', bounds $=(0, \mathrm{sqrt}(12))$, integer=False,

rules $=$ PiecewiseLinear $($ values $=[\mathrm{np} . \mathbf{s q r t}(\mathrm{CN})$ for $\mathrm{CN}$ in range $(0,13)]$,

0021.eps enlarge to full single column

$$
\begin{aligned}
& \text { breakpoints }=[\mathrm{CN} \text { for } \mathrm{CN} \text { in range } \\
& \quad(0,13)] \\
& \text { input_desc }=\mathrm{m} . \mathrm{Ci}))
\end{aligned}
$$

686 Next, we introduce the descriptor for the normalized 687 cohesive energy. Here, we utilize the number of atoms in the 688 cluster, $\mathrm{N}$, and the square root of the bulk coordination 689 number (i.e., $\sqrt{12}$ ) as a normalizing coefficient. Since this 690 descriptor is a single scalar quantity, we invoke the 691 addGlobalDescriptor method.

\footnotetext{
14 m.addGlobalDescriptor ('Ecoh',
} rules=EqualTo $($ SumSites $($ desc $=m$. CNRi

0022.eps er enlarge to full single column

692 Finally, we introduce a descriptor for the size of the 693 nanocluster. Note how, by setting the lower and upper bounds 694 on the descriptor to be equal, we essentially constrain the 695 nanocluster to be of a specific size.

$$
\begin{array}{c|l}
\text { m. addGlobalDescriptor ('Size', bounds=(N,N), } & \begin{array}{l}
\text { 0023.eps } \\
\text { enlarge so the font } \\
\text { rules=EqualTo(SumSites (desc=m.Yi) }
\end{array} \\
\text { matches others }
\end{array}
$$

At this point, the feasible space for the optimization model is 696 fully specified. We finalize the model and instruct MatOpt to 697 solve it by specifying an objective. In this example, the 698 objective to be maximized will be the previously defined (code 699 line 14) descriptor m. Ecoh, noting that any Expression 700 object can be used to that purpose, in general. The 701 maximize function will formulate an undelying Pyomo 702 model object and call the appropriate optimization solver 703 routine.

$19 \quad \mathrm{D}=\mathrm{m} \cdot \operatorname{maximize}(\mathrm{m} . \mathrm{Ecoh})$

In this specific example, MatOpt will generate a model with 705 5292 variables (1914 continuous, 3231 binary, 147 integer) 706 and 8079 constraints ( 7488 inequalities, 591 equalities). Upon 707 testing with our default optimization solver, CPLEX 12.10, on 708 an 8-core Intel i7 laptop, the model could be solved to global 709 optimality (i.e., relative and absolute gap tolerances set to 710 zero) in approximately $33 \mathrm{~s}$. The solution attains a globally 711 optimal normalized cohesive energy of 0.707 and is depicted in 712 Figure 8a.

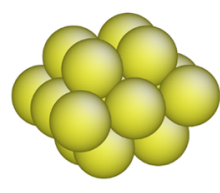

(a) $N=17$

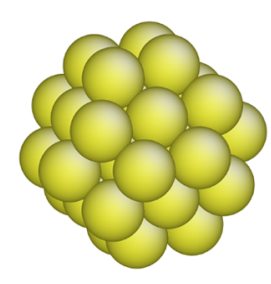

(b) $N=34$

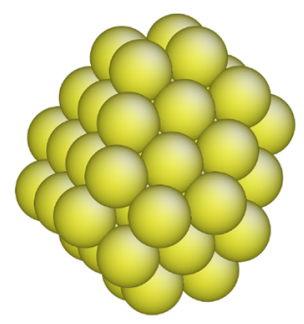

(c) $N=55$
Figure 8. Example optimally cohesive monometallic nanoclusters.

We note that the size of the model depends on the size of 714 the canvas (code line 6). More specifically, if we use four shells 715 of FCC lattice locations around the origin (309 sites) instead 716 of three shells (147 sites), we will obtain a model with 11322717 variables and 17583 constraints. The model will grow to 718 20790 variables and 32631 constraints when using 5 shells 719 (561 sites) and to 34476 variables and 54503 constraints, 720 when usino 6 shells ( 923 cites) Whereas a laroer canvas 721 after enlarging make sure has 722 0017-0025 are the same font $\begin{aligned} & \text { ore } 723 \\ & \text { our } 724 \\ & \text { bal } 725\end{aligned}$ size

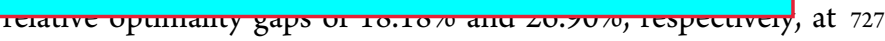
the $1 \mathrm{~h}$ time limit. We should highlight, however, that 728 increasing the canvas size in this case would not be justified, as 729 it would have no impact on the ability of the tool to identify 730 the optimal solution.

731

If the optimization solver is successful, a Design object is 732 returned that can be written to standard material file formats. 733 Here, we create a Protein Data Bank (PDB) file, ${ }^{47}$ noting that 734 additional parsers are also implemented and can be used as 735 necessary.

While the sample code presented above is intentionally terse, 737 there are many ways to modify the script to collect and analyze 738 a variety of results. The most obvious extension is to place the 739 


$$
20 \text { if( } D \text { is not None): }
$$

$$
21 \text { D.toPDB ('result_\{\}.pdb'. } \operatorname{format}(N) \text { ) }
$$

740 model generation and solution in a Python loop to generate 741 designs across a range of sizes $N$. In Figure 8, we present a few 742 example results from solving the above simple model within 743 such a loop. Here, we should remark that, although the 744 parameter $N$ may impact the solution times from imposing 745 various inherent combinatorial complexities, it does not 746 directly impact the size of the model, and since we used 747 same size canvases in all those examples, the computational 748 times required to solve them were all in the same order of 749 magnitude (around $100 \mathrm{~s}$ ).

750 The code can be further tailored by changing the 751 contributions to cohesive energy with specially regressed 752 coefficients to better represent a metal of interest, as presented 753 in the work of Isenberg et al. ${ }^{28}$

754 Before we conclude this case study, we note that, in addition 755 to the basic mathematical optimization model presented above, 756 the original work of Isenberg et al. ${ }^{28}$ made several customized 757 problem-specific modifications to improve the optimization 758 model's tractability and the interpretability of its solution. One 759 major such modification was the introduction of a set of 760 symmetry-breaking constraints to eliminate certain equivalent 761 feasible solutions (i.e., nanostructures symmetric to others via 762 rotation and reflection) while making sure that at least one 763 representative nanostructure remained feasible. We note that 764 symmetry-breaking constraints are typically problem-specific, 765 depending strongly on the applicable lattice topology. As a 766 general-purpose set of symmetry breaking constraints is hard 767 to devise, MatOpt does not automatically apply such 768 constraints. Here, it should be emphasized that the lack of 769 symmetry-breaking constraints will not affect the optimality of 770 the obtained solutions, rather the solutions obtained by 771 MatOpt will indeed be optimal albeit in an arbitrary 772 orientation. In any case, a user could in principle define 773 additional descriptors and rules to formulate symmetry774 breaking constraints on an ad-hoc basis. However, it is always 775 advisable that such action be empirically investigated on a case776 by-case basis, as declaring symmetry-breaking constraints may 777 not necessarily result in the most efficient MILP model 778 formulation and thus may not help with overall tractability.

779 Perovskite Dopant Design. Hanselman et al. ${ }^{27}$ proposed 780 a mathematical optimization based framework for designing 781 doped perovskites. Their study identified the optimal atomic782 scale patterns of In dopants at the $\mathrm{B}$-sites in $\mathrm{BaFe}_{1-x} \mathrm{In}_{x} \mathrm{O}_{3-\delta}$ 783 perovskites that achieve the highest oxygen diffusivity, an 784 important property for such perovskites to be used as oxygen 785 carriers in a chemical looping system. The optimization model 786 proposed in the aforementioned study identified oxygen excess 787 energy as the target functionality of interest, decomposing it 788 into contributions from conformations associated with each 789 oxygen atom in the lattice. More specifically, the paper 790 investigated oxygen atom conformations that consisted of 10 791 nearest B-sites and represented all possible ways of placing 792 between zero to five dopants around a particular oxygen atom. 793 The set of 74 rotationally unique conformations, so-called 794 "motifs," was then identified and their oxygen excess energies 795 were evaluated via DFT calculations. The 74 evaluations 796 formed a structure-function relationship that was then 797 encoded into an MILP model that attempted to maximize 798 the number of oxygen sites exhibiting lowest excess energies.
We highlight how this conformation based encoding is another 799 way of expressing nonlinear structure-function relationships 800 via linear constraints that utilize binary variables.

The above approach and model can be readily reproduced 802 using MatOpt. The full implementation is provided in Section 803 B of the Supporting Information, while below we highlight 804 only some key aspects. To implement this optimization model 805 using MatOpt, we first construct a PerovskiteLattice 806 object to hold perovskite-specific lattice site information. We 807 confine our optimization to a periodic supercell using a 808 Shape object, which defines the geometry and size of the 809 supercell, in conjunction with a Tiling object to handle 810 boundary neighbor relationships. In particular, we create a 811 rectangular prism-shaped supercell Canvas with periodicity 812 in all three dimensions using RectPrism and CubicTil- 813 ing objects, which constitute available specializations of the 814 Shape and Tiling classes, respectively. Here, we note a 815 small trick to shift the shape slightly to avoid duplication of 816 sites on the border of the periodic cell, which is necessary to do 817 for proper visualization of the canvas. We highlight that such 818 shift has no impact on solution optimality and is in fact not 819 necessary for the correctness of the canvas itself. The 820 visualization of this perovskite canvas can be found in Figure 821 f 9 9a. Next, we load motifs from standard material file formats, $822 \mathrm{f} 9$

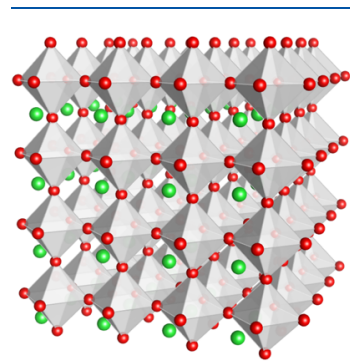

(a)

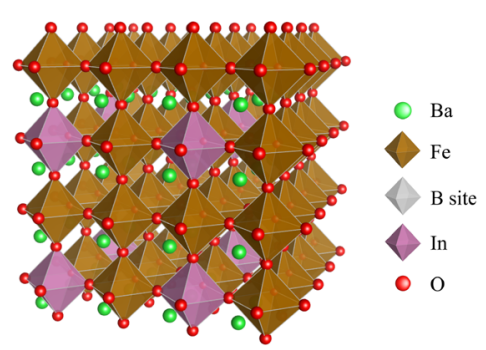

(b)
Figure 9. (a) Doped perovskite design canvas and (b) optimal design that maximizes the number of sites displaying one of the three motifs with lowest oxygen excess energy.

noting that parsers for several common formats such as PDB, 823 POSCAR, and XYZ are available. We then initialize a 824 MatOptModel object from the defined conformations and 825 sites. Note how MatOpt will automatically encode basic 826 variables and constraints to indicate those conformations (eqs 827 22-25). To specify additional constraints in the model, we 828 create several descriptors for the activity, local dopant 829 concentration, and the global dopant concentration. We 830 specify these descriptors over different subsets of sites and 831 atom types. For example, the activity descriptor is defined over 832 the oxygen sites only, while the dopant budgets are expressed 833 only over B-sites. Note the use of the addSitesTypesDe- 834 scriptor and addGlobalTypesDescriptor meth- 835 ods, as appropriate.

In this case study, we shall specify our objective as the 837 maximization of the number of sites displaying conformations 838 with the lowest three oxygen excess energies among all possible 839 conformations. We note, however, that additional objectives 840 stated in the original work can also be modeled and solved via 841 MatOpt by simply changing parameters of the activity 842 descriptor. We specifically consider a unit cell with four 843 atoms on the edge, which corresponds to the size chosen by 844 Hanselman et al. ${ }^{27}$ who carefully investigated the trade-off 845 

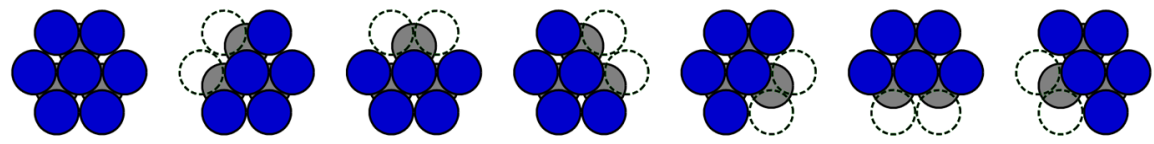

Figure 10. Material conformations relevant for the bifunctional catalyst example. The gray circles represent an extended $\{111\}$ layer of Pt atoms while the blue circles represent the (patchy) overlayer of $\mathrm{Ni}$ atoms. Dashed circles represent missing Ni atoms, resulting in reactive step sites next to the central $\mathrm{Ni}$ atom.

846 between solution optimality and model tractability in this 847 setting. The MatOpt-generated model consists of 6723 848 variables (195 continuous, 6528 binary) and 16259 con849 straints (16 000 inequalities, 259 equalities) and can be solved 850 to global optimality (using similar zero gap tolerances and 851 machine as before) in approximately $33 \mathrm{~min}$. The global 852 optimal solution is shown in Figure 9b. It features an objective 853 value of 0.5 and perfectly reproduces the original result from 854 Hanselman et al. ${ }^{27}$

855 Before we conclude, we highlight that the code provided in 856 the Supporting Information constitutes an example MatOpt 857 implementation for conformation based optimization models. 858 Starting from this code as the basis, a user may adapt the 859 definition of motifs and the way in which properties of interest 860 are calculated from first-principles, and then rely on MatOpt to 861 automatically instantiate and solve the rigorous mathematical 862 optimization model that searches for the best combinations of 863 motifs in the design space.

864 Bifunctional Catalyst Design. To further demonstrate 865 the capabilities of the MatOpt toolkit, we present here a surface 866 design problem inspired by the recent work of Núnez and 867 Vlachos. ${ }^{44}$ In that work, the authors presented a machine868 learned model for predicting surface reactivity of ammonia 869 decomposition on a patchy $\mathrm{Ni}-\mathrm{Pt}$ bifunctional catalyst and 870 then designed a surface by applying a simulated annealing 871 optimization algorithm. While their approach is tailored to 872 make accurate predictions of reactivity, the use of simulated 873 annealing lacks guarantees of producing the globally optimal 874 design. In this example, we present a complementary MILP 875 based approach that can identify provably optimal structures 876 for a simplified version of the relevant chemistry.

877 This design problem calls for identifying the optimal 878 patterning of a single layer of $\mathrm{Ni}$ atoms on top of a $\mathrm{Pt}\{111\}$ 879 surface. The placement of $\mathrm{Ni}$ atoms creates facet and edge sites 880 that contribute to the turnover of the ammonia decomposition 881 reaction. As a first order approximation of the microkinetic 882 model presented in the work of Núñez and Vlachos, ${ }^{44}$ we shall 883 simply assume that the slab reactivity is proportional to the 884 sum of pairs of edge sites immediately next to a facet site. This 885 simplified viewpoint neglects contributions to reactivity from 886 adsorbates that take a longer path diffusing across the catalyst 887 surface between sites further away than nearest neighbors. 888 However, we remark that more complicated contributions 889 could be conceptually represented (and encoded in MatOpt) 890 via additional variables, constraints, and parameters for the 891 turnover on the catalyst surface.

892 The full MatOpt implementation of this design problem can 893 be found in section C of the Supporting Information. To 894 design a periodic surface, we create a Parallelepiped 895 shaped canvas with periodicity defined by PlanarTiling. 896 We again follow a conformation based modeling strategy to 897 encode nonlinear structure-function relationships and utilize $898 \mathrm{MatOpt}$ 's capabilities to indicate identified conformations 899 automatically. For reference, we plot in Figure 10 the seven relevant conformations, including an FCC $\{111\}$ facet site and 900 six orientations of edge sites.

901

As a simplified model of catalyst turnover, we consider here 902 that the combination of a facet site next to an edge site gives 903 rise to an "ideal" reactive step site. To represent this in our 904 optimization model, we define additional material descriptors 905 to indicate the conjunction of a conformation of type "A" (i.e., 906 a facet site) next to conformation of type "B" (i.e., an edge 907 site). We then fix the possible chemical identity of the various 908 layers of the design. Additionally, we can improve the 909 tractability of the model by arbitrarily placing one 910 conformation on the surface, breaking symmetry and 911 simplifying the design space.

912

Furthermore, we model the surface energy of the resulting 913 patterns with the goal of mapping the Pareto-optimal frontier 914 of activity against stability in this design space. To obtain the 915 surface energy, we first employ the formation energy model 916 proposed for this system in Núñez et al. ${ }^{23}$ In this model, each 917 atom contributes to lowering the formation energy in a manner 918 that is proportional to the square root of its coordination 919 number; that is, lower coordinated atoms contribute to a 920 higher formation energy. More specifically, eq 38 presents the 921 descriptor for the formation energy of the slab (normalized by 922 the cohesive energy of the metal), $\hat{E}^{\text {form }}$, as a function of 923 coordination numbers $C_{i}$ at each site $i$. The surface energy is 924 then approximated in eq 39 by dividing the formation energy 925 with the surface area, where as a proxy for the latter we use the 926 square of the number of atoms on the edge of the canvas, $N_{\text {edge. }} 927$ Note here that, since we are using a fixed size periodic canvas 928 during each run, we will identify the same optimal results when 929 using surface energy or formation energy as the stability 930 indicator. After some experimentation using differently sized 931 periodic tiles, in order to explore the impact of periodicity 932 limitations on the obtained designs as well as the tractability of 933 the resulting models, we chose to work with tiles of size $8 \times 8934$ atoms, which were found to be a good compromise inasmuch 935 as they were generally tractable and did not show solution 936 differences compared to using $9 \times 9$ atom tiles.

937

$$
\begin{aligned}
& \hat{E}^{\text {form }}=\sum_{i \in I}\left(1-\sqrt{\frac{C_{i}}{C N_{\text {bulk }}}}\right) \\
& \hat{E}^{\text {surf }}=\hat{E}^{\text {form }} / N_{\text {edge }}^{2}
\end{aligned}
$$

Note how the values of the per-atom contributions to the 940 surface energy have been normalized so that they all lie 941 between 0 and 1 , with a value of 0 corresponding to bulk 942 atoms that do not contribute to the surface energy and a value 943 of 1 corresponding at the limit to the hypothetical contribution 944 of an isolated atom that would have increased the surface 945 energy the most. In calculating surface energy via the above 946 method, we exclude sites in the bottom two layers of the 947 canvas, which are fixed to be fully occupied with Pt atoms. 948 
949 To build a Pareto frontier of activity against stability of such 950 nanostructured surfaces, we perform a loop to solve the model 951 for a range of thresholds on the surface activity (i.e., lower 952 bound on the number of active sites). The resulting frontier is 953 presented in Figure 11. We note that each frontier point

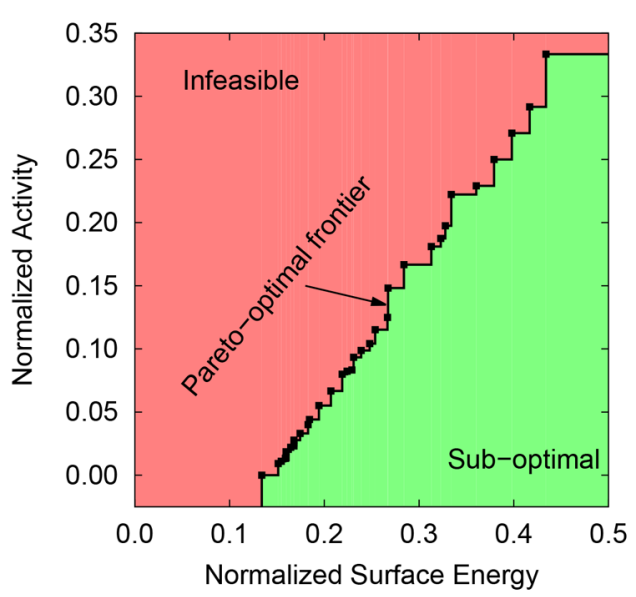

Figure 11. Pareto-optimal frontier for the bifunctional catalyst design example.

954 represents the optimal solution of a separate MatOpt-generated 955 optimization model, each featuring 10240 variables (1667 956 continuous, 8189 binary, 384 integer) and 38659 constraints 957 (37376 inequalities and 1283 equalities). On average, these 958 models took approximately $410 \mathrm{~s}$ to solve to global optimality 959 (zero gap tolerance) using the same computational setup as the 960 previous case studies.

961 A few representative Pareto-optimal solutions identified are 962 plotted in Figure 12. We observe that nanostructuring frequent 963 grooves on the Ni layer yields a larger density of edge sites and, 964 hence, increases activity. However, it has a negative effect on 965 overall stability, which is promoted when there are fewer 966 grooves. A middle ground can be found when atoms are placed 967 intermittently as bridges that disrupt the grooves, creating a 968 stabilizing effect without significant reduction in activity. 969 Finally, it is worth noting that the maximally active design 970 was equivalent to the design proposed by Núñez and 971 Vlachos, ${ }^{44}$ suggesting that, at least to a first approximation, 972 their identified design was in fact optimally active.

\section{$973 \square$ CONCLUSIONS}

974 In this paper, we presented a general-purpose approach for 975 designing nanostructured materials. For this, we compiled a set 976 of shared features of nanomaterial design problems and then 977 developed modular variables and constraints to represent the basic features of their respective optimization models. To 978 enable wider adoption of this materials design paradigm, we 979 have created MatOpt, a Python module that offers object- 980 oriented classes for specifying materials information and for 981 casting mathematical optimization models for their nano- 982 structured design. This paper introduced this software and 983 demonstrated its usage via three examples. A detailed example 984 of the module applied to a nanocluster cohesive energy 985 minimization problem illustrated the basic syntax and logic for 986 creating models. The perovskite dopant optimization model 987 illustrated how to handle periodicity and utilize data from DFT 988 calculations in the context of this optimization task. Finally, the 989 implementation of a bifunctional catalyst surface design model 990 illustrated more complex features and demonstrated the facile 991 generation of Pareto-optimal results. MatOpt is distributed as 992 part of the IDAES software distribution, and we hope that it 993 shall enable a rigorous mathematical optimization paradigm in 994 the domain of nanostructured materials design.

\section{DATA AND SOFTWARE AVAILABILITY}

995

The software MatOpt is freely available at https://github.com/ 997 IDAES/idaes-pse/tree/main/idaes/apps/matopt. All data re- 998 quired to run the case studies presented in this paper is 999 included within the code provided in sections B and C of the 1000 Supporting Information.

\section{ASSOCIATED CONTENT}

The Supporting Information is available free of charge at 1004 https://pubs.acs.org/doi/10.1021/acs.jcim.1c00984.

Additional information about some of MatOpt's key 1006 modeling objects as well as code listings for the 1007 perovskite design problem and the bifunctional catalyst 1008 design problem (PDF)

\section{AUTHOR INFORMATION}

\section{Corresponding Author}

Chrysanthos E. Gounaris - Department of Chemical Engineering, Carnegie Mellon University, Pittsburgh, Pennsylvania 15213, United States; 1 orcid.org/00000001-5779-2510; Email: gounaris@cmu.edu

\section{Authors}

Christopher L. Hanselman - Department of Chemical Engineering, Carnegie Mellon University, Pittsburgh, Pennsylvania 15213, United States

1017

Xiangyu Yin - Department of Chemical Engineering, Carnegie 1020 Mellon University, Pittsburgh, Pennsylvania 15213, United 1021 States; @ orcid.org/0000-0003-2868-1728

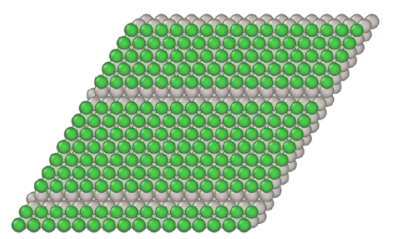

$\mathrm{A}=0.166$

$\mathrm{E}=0.284$

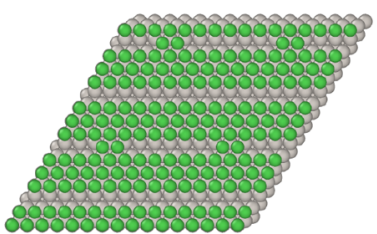

$\mathrm{A}=0.271$

$\mathrm{E}=0.398$

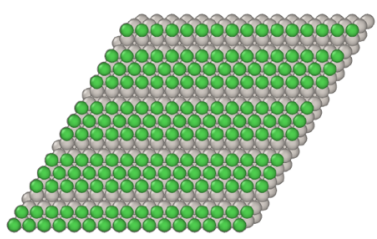

$\mathrm{A}=0.333$

$\mathrm{E}=0.434$

Figure 12. Example Pareto-optimal results for the Ni/Pt $\{111\}$ bifunctional catalyst example. Normalized activity (A) and surface energy (E) values are provided below each tile. 
1023

1024

1025

David C. Miller - National Energy Technology Laboratory, Pittsburgh, Pennsylvania 15236, United States; (1) orcid.org/ 0000-0002-7378-5625

1026 Complete contact information is available at:

1027 https://pubs.acs.org/10.1021/acs.jcim.1c00984

\section{Notes}

1029 This paper was prepared as an account of work sponsored by 1030 an agency of the United States Government. Neither the 1031 United States Government nor any agency thereof, nor any of 1032 their employees, makes any warranty, express or implied, or 1033 assumes any legal liability or responsibility for the accuracy, 1034 completeness, or usefulness of any information, apparatus, 1035 product, or process disclosed, or represents that its use would 1036 not infringe privately owned rights. Reference herein to any 1037 specific commercial product, process, or service by trade name, 1038 trademark, manufacturer, or otherwise does not necessarily 1039 constitute or imply its endorsement, recommendation, or 1040 favoring by the United States Government or any agency 1041 thereof. The views and opinions of authors expressed herein do 1042 not necessarily state or reflect those of the United States 1043 Government or any agency thereof.

1044 The authors declare no competing financial interest.

\section{ACKNOWLEDGMENTS}

1046 This work was conducted as part of the Institute for the Design 1047 of Advanced Energy Systems (IDAES) with support through 1048 the Simulation-Based Engineering, Crosscutting Research 1049 Program within the U.S. Department of Energy's Office of 1050 Fossil Energy and Carbon Management.

\section{REFERENCES}

1052 (1) Barth, J. V.; Costantini, G.; Kern, K. Engineering atomic and 1053 molecular nanostructures at surfaces. Nature 2005, 437, 671.

1054 (2) Xia, Y.; Xiong, Y.; Lim, B.; Skrabalak, S. E. Shape-controlled 1055 synthesis of metal nanocrystals: simple chemistry meets complex 1056 physics? Angew. Chem. 2009, 48, 60-103.

1057 (3) Xia, Y.; Xia, X.; Peng, H.-C. Shape-controlled synthesis of 1058 colloidal metal nanocrystals: thermodynamic versus kinetic products. 1059 J. Am. Chem. Soc. 2015, 137, 7947-7966.

1060 (4) Satyavolu, N. S. R.; Loh, K. Y.; Tan, L. H.; Lu, Y. Discovery of 1061 and insights into DNA "codes" for tunable morphologies of metal 1062 nanoparticles. Small 2019, 15, 1900975.

1063 (5) King, M. E.; Personick, M. L. Defects by design: synthesis of 1064 palladium nanoparticles with extended twin defects and corrugated 1065 surfaces. Nanoscale 2017, 9, 17914-17921.

1066 (6) Park, J.; Kwon, T.; Kim, J.; Jin, H.; Kim, H. Y.; Kim, B.; Joo, S. $1067 \mathrm{H}$.; Lee, K. Hollow nanoparticles as emerging electrocatalysts for 1068 renewable energy conversion reactions. Chem. Soc. Rev. 2018, 47, 1069 8173-8202.

1070 (7) Nørskov, J. K.; Bligaard, T.; Rossmeisl, J.; Christensen, C. H. 1071 Towards the computational design of solid catalysts. Nat. Chem. 2009, 1072 1, 37-46.

1073 (8) Maurer, R. J.; Freysoldt, C.; Reilly, A. M.; Brandenburg, J. G.; 1074 Hofmann, O. T.; Björkman, T.; Lebègue, S.; Tkatchenko, A. Advances 1075 in density-functional calculations for materials modeling. Annu. Rev. 1076 Mater. Res. 2019, 49, 1-30.

1077 (9) Garlyyev, B.; Liang, Y.; Xue, S.; Watzele, S.; Fichtner, J.; Li, W.1078 J.; Ding, X.; Bandarenka, A. S. Theoretical and experimental 1079 identification of active electrocatalytic surface sites. Curr. Opin. 1080 Electrochem. 2019, 14, 206-213.

1081 (10) Jain, A.; Ong, S. P.; Hautier, G.; Chen, W.; Richards, W. D.; 1082 Dacek, S.; Cholia, S.; Gunter, D.; Skinner, D.; Ceder, G.; Persson, K. 1083 A. Commentary: the Materials Project: a materials genome approach 1084 to accelerating materials innovation. APL Mater. 2013, 1, 011002.
(11) Saal, J. E.; Kirklin, S.; Aykol, M.; Meredig, B.; Wolverton, C. 1085 Materials design and discovery with high-throughput density 1086 functional theory: the open quantum materials database (OQMD). 1087 JOM 2013, 65, 1501-1509.

1088

(12) Curtarolo, S.; Setyawan, W.; Hart, G. L.; Jahnatek, M.; 1089 Chepulskii, R. V.; Taylor, R. H.; Wang, S.; Xue, J.; Yang, K.; Levy, O.; 1090 Mehl, M. J.; Stokes, H. T.; Demchenko, D. O.; Morgan, D. AFLOW: 1091 An automatic framework for high-throughput materials discovery. 1092 Comput. Mater. Sci. 2012, 58, 218-226.

1093

(13) Liu, Y.; Zhao, T.; Ju, W.; Shi, S. Materials discovery and design 1094 using machine learning. J. Materiomics 2017, 3, 159-177. 1095

(14) Ulissi, Z. W.; Singh, A. R.; Tsai, C.; Nørskov, J. K. Automated 1096 discovery and construction of surface phase diagrams using machine 1097 learning. J. Phys. Chem. Lett. 2016, 7, 3931-3935.

(15) Ulissi, Z. W.; Medford, A. J.; Bligaard, T.; Nørskov, J. K. To 1099 address surface reaction network complexity using scaling relations 1100 machine learning and DFT calculations. Nat. Commun. 2017, 8, 1-7. 1101

(16) Ulissi, Z. W.; Tang, M. T.; Xiao, J.; Liu, X.; Torelli, D. A.; 1102 Karamad, M.; Cummins, K.; Hahn, C.; Lewis, N. S.; Jaramillo, T. F.; 1103 Chan, K.; Nørskov, J. K. Machine-learning methods enable exhaustive 1104 searches for active bimetallic facets and reveal active site motifs for 1105 CO2 reduction. ACS Catal. 2017, 7, 6600-6608. 1106

(17) Goldsmith, B. R.; Esterhuizen, J.; Liu, J.-X.; Bartel, C. J.; Sutton, 1107 C. A. Machine learning for heterogeneous catalyst design and 1108 discovery. AIChE J. 2018, 64, 2311-2323.

1109

(18) Tran, K.; Palizhati, A.; Back, S.; Ulissi, Z. W. Dynamic 1110 workflows for routine materials discovery in surface science. J. Chem. 1111 Inf. Model. 2018, 58, 2392-2400.

1112

(19) Calle-Vallejo, F.; Martínez, J. I.; García-Lastra, J. M.; Sautet, P.; 1113 Loffreda, D. Fast prediction of adsorption properties for platinum 1114 nanocatalysts with generalized coordination numbers. Angew. Chem. 1115 2014, 53, 8316-8319.

1116

(20) Calle-Vallejo, F.; Loffreda, D.; Koper, M. T.; Sautet, P. 1117 Introducing structural sensitivity into adsorption-energy scaling 1118 relations by means of coordination numbers. Nat. Chem. 2015, 7, 1119 403-410.

1120

(21) Calle-Vallejo, F.; Tymoczko, J.; Colic, V.; Vu, Q. H.; Pohl, M. 1121 D.; Morgenstern, K.; Loffreda, D.; Sautet, P.; Schuhmann, W.; 1122 Bandarenka, A. S. Finding optimal surface sites on heterogeneous 1123 catalysts by counting nearest neighbors. Science 2015, 350, 185-189. 1124

(22) Rück, M.; Bandarenka, A.; Calle-Vallejo, F.; Gagliardi, A. Fast 1125 identification of optimal pure platinum nanoparticle shapes and sizes 1126 for efficient oxygen electroreduction. Nanoscale Adv. 2019, 1, 2901- 1127 2909.

1128

(23) Núñez, M.; Lansford, J.; Vlachos, D. Optimization of the facet 1129 structure of transition-metal catalysts applied to the oxygen reduction 1130 reaction. Nat. Chem. 2019, 11, 449-456.

1131

(24) Hanselman, C. L.; Gounaris, C. E. A mathematical optimization 1132 framework for the design of nanopatterned surfaces. AIChE J. 2016, 1133 $62,3250-3263$.

1134

(25) Hanselman, C. L.; Zhong, W.; Tran, K.; Ulissi, Z. W.; Gounaris, 1135 C. E. Optimization-based design of active and stable nanostructured 1136 surfaces. J. Phys. Chem. C 2019, 123, 29209-29218.

1137

(26) Hanselman, C. L.; Tafen, D. N.; Alfonso, D. R.; Lekse, J. W.; 1138 Matranga, C.; Miller, D. C.; Gounaris, C. E. Design of Doped 1139 Perovskite Oxygen Carriers Using Mathematical Optimization. 1140 Comput.-Aided Chem. Eng. 2018, 44, 2461-2466.

1141

(27) Hanselman, C. L.; Tafen, D. N.; Alfonso, D. R.; Lekse, J. W.; 1142 Matranga, C.; Miller, D. C.; Gounaris, C. E. A framework for 1143 optimizing oxygen vacancy formation in doped perovskites. Comput. 1144 Chem. Eng. 2019, 126, 168-177.

1145

(28) Isenberg, N. M.; Taylor, M. G.; Yan, Z.; Hanselman, C. L.; 1146 Mpourmpakis, G.; Gounaris, C. E. Identification of optimally stable 1147 nanocluster geometries via mathematical optimization and density- 1148 functional theory. Mol. Sys. Des. Eng. 2020, 5, 232-244. 1149

(29) Yin, X.; Isenberg, N. M.; Hanselman, C. L.; Dean, J. R.; 1150 Mpourmpakis, G.; Gounaris, C. E. Designing stable bimetallic 1151 nanoclusters via an iterative two-step optimization approach. Mol. 1152 Sys. Des. Eng. 2021, 6, 545-557. 
1154 (30) Yin, X.; Gounaris, C. E. Search methods for inorganic materials 1155 crystal structure prediction. Curr. Opin. Chem. Eng. 2022, 35, 100726. 1156 (31) Goronzy, D. P.; Ebrahimi, M.; Rosei, F.; Arramel; Fang, Y.; De 1157 Feyter, S.; Tait, S. L.; Wang, C.; Beton, P. H.; Wee, A. T.; Weiss, P. S.; 1158 Perepichka, D. F. Supramolecular assemblies on surfaces: Nano1159 patterning, functionality, and reactivity. ACS Nano 2018, 12, 744511607481.

1161 (32) National Energy Technology Laboratory, Institute for the 1162 Design of Advanced Energy Systems (IDAES). www.idaes.org/ 1163 download/.

1164 (33) IDAES PSE framework documentation. https://idaes-pse. 1165 readthedocs.io/en/stable/getting_started/index.html.

1166 (34) MatOpt documentation. https://idaes-pse.readthedocs.io/en/ 1167 stable/user_guide/modeling_extensions/matopt/index.html.

1168 (35) MatOpt use cases. https://idaes.github.io/examples-pse/latest/ 1169 Examples/MatOpt/index.html.

1170 (36) Gropp, W.; Moré, J. J. Optimization Environments and the 1171 NEOS Server. Approximation Theory and Optimization, Buhman, M. 1172 D., Iserles, A., Eds.; Cambridge University Press, 1997; pp 167-182. 1173 (37) Czyzyk, J.; Mesnier, M. P.; Moré, J. J. The NEOS server. IEEE 1174 Comput. Sci. Eng. 1998, 5, 68-75.

1175 (38) Dolan, E. D. NEOS Server 4.0 Administrative Guide. arXiv.org, 1176 2001, cs/0107034. https://arxiv.org/abs/cs/0107034.

1177 (39) Larsen, A. H.; Mortensen, J. J.; Blomqvist, J.; Castelli, I. E.; 1178 Christensen, R.; Dułak, M.; Friis, J.; Groves, M. N.; Hammer, B.; 1179 Hargus, C.; Hermes, E. D.; Jennings, P. C.; Jensen, P. B.; Kermode, J.; 1180 Kitchin, J. R.; Kolsbjerg, E. L.; Kubal, J.; Kaasbjerg, K.; Lysgaard, S.; 1181 Maronsson, J. B.; Maxson, T.; Olsen, T.; Pastewka, L.; Peterson, A.; 1182 Rostgaard, C.; Schiotz, J.; Schutt, O.; Strange, M.; Thygesen, K. S.; 1183 Vegge, T.; Vilhelmsen, L.; Walter, M.; Zeng, Z.; Jacobsen, K. W. The 1184 atomic simulation environment-a Python library for working with 1185 atoms. J. Phys.: Condens. Matter 2017, 29, 273002.

1186 (40) Ong, S. P.; Richards, W. D.; Jain, A.; Hautier, G.; Kocher, M.; 1187 Cholia, S.; Gunter, D.; Chevrier, V. L.; Persson, K. A.; Ceder, G. 1188 Python Materials Genomics (PyMatGen): a robust, open-source 1189 python library for materials analysis. Comput. Mater. Sci. 2013, 68, 1190 314-319.

1191 (41) Plimpton, S. Fast parallel algorithms for short-range molecular 1192 dynamics. J. Comput. Phys. 1995, 117, 1-19.

1193 (42) Hart, W. E.; Watson, J.-P.; Woodruff, D. L. Pyomo: modeling 1194 and solving mathematical programs in Python. Math. Program. 2011, 1195 3, 219-260.

1196 (43) Hart, W. E.; Laird, C. D.; Watson, J.-P.; Woodruff, D. L.; 1197 Hackebeil, G. A.; Nicholson, B. L.; Siirola, J. D. Pyomo-optimization 1198 modeling in python; Springer, 2017; Vol. 67.

1199 (44) Núñez, M.; Vlachos, D. G. Multiscale modeling combined with 1200 active learning for microstructure optimization of bifunctional 1201 catalysts. Ind. Eng. Chem. Res. 2019, 58, 6146-6154.

1202 (45) Tomanek, D.; Mukherjee, S.; Bennemann, K. Simple theory for 1203 the electronic and atomic structure of small clusters. Phys. Rev. B 1204 1983, 28, 665 .

1205 (46) Oliphant, T. E. A guide to NumPy; Trelgol Publishing USA, 1206 2006; Vol. 1.

1207 (47) Berman, H. M.; Westbrook, J.; Feng, Z.; Gilliland, G.; Bhat, T. 1208 N.; Weissig, H.; Shindyalov, I. N.; Bourne, P. E. The protein data 1209 bank. Nucleic Acids Res. 2000, 28, 235-242. 